\title{
A Context-Awareness Architecture for Managing Thermal Energy in an nZEB Building
}

\author{
Oscar Hernández Uribe \\ Information Technology, Electronics, and Control \\ CIATEQ AC \\ Santiago de Querétaro \\ oscar.hernandez@ciateq.mx
}

\author{
Matilde Santos \\ Computer Architecture and Automatic Control \\ UCM \\ Madrid, Spain \\ msantos@ucm.es
}

\author{
María C. Garcia-Alegre/Domingo Guinea \\ Renewable Energy, Fuel Cell and Hydrogen Laboratory (LERH) \\ Center for Automation and Robotics (CAR), CSIC-UPM \\ Arganda, Madrid, Spain \\ mcarmen.garcia-alegre@csic.es, domingo.guinea@csic.es
}

\begin{abstract}
Global warming is seriously affecting society, but also to a lesser extent the longevity of the population and migration of people from the countryside to the cities present challenges for governments. Smart applications (smart city, smart grid, smart buildings, smart water, smart health) offer an alternative to deal with these challenges. Pervasive sensors, with increasingly powerful features, allow innovative developments, making possible a progressive growth in the so-called smart applications. Because information is the backbone of any type of smart environment, this work presents an architecture approach to take advantage of the capabilities of advanced sensors. Specifically in this work this architecture is applied to renewable energy systems for a better management of the thermal energy. A semantic sensors network has been developed to provide context awareness for managing thermal flow in a near Zero Energy Building (nZEB). The key elements are an Ontology Web Language (OWL) to describe the sensors and contextual knowledge, and a Semantic Web Rule Language (SWRL) to represent rule-based inferences for context reasoning. The main goal is to improve thermal energy comfort in a building with a reduction in the energy used.
\end{abstract}

Keywords—smart energy; control architecture; web ontology; context-awareness; semantic sensor; $n Z E B$ building.

\section{INTRODUCTION}

The building sector has the highest energy consumption and also the fastest rate of growth. Indoor heating and cooling needs are the most energy consuming demands in buildings. Although solar energy would be sufficient to meet the demands of thermal comfort in low and mid-latitude regions, and it is also the most widely used worldwide [1], unfortunately this energy source has strong limitations such as discontinuity and large variations depending on the weather, the season or time of the day. So it would be necessary to have Thermal Energy Storage System (TESS) for the end user at any time.

Sensible heat energy storage systems (SHESS) such as the low enthalpy geothermal energy have attracted enormous interest in recent years in order to save energy and reduce $\mathrm{CO} 2$ emissions [2-3]. Governments define new policies for construction, where it is intended that the buildings are certified as near Zero Energy Buildings (nZEB) or Zero Energy Buildings (ZEB), or even be energy suppliers [4-5]. These challenges drive efficient management of energy resources, the use of renewable energy for heating and cooling, energy storage systems, and improvements in the building envelope. Challenges directly related to the development of a country.

Moreover, today the Internet is present in virtually all aspects of our life and the Semantic Web technologies open the possibility of creating a network of intelligible data for any type of electronic device [6]. Sensor networks have benefited from development of standards and technologies of the World Wide Web Consortium (W3C). For example, the Semantic Sensor Networks Incubator Group (SSN-XG) has developed a sensor called Semantic Sensor Network Ontology (SSNO) ${ }^{1}$. There exist a variety of intelligent applications in which the use of semantic web technologies and ontologies are the core of the solutions of problems related to user context, energy, building information or the integration of renewable energy systems [712]. In a couple of decades the population will be interconnected through networks of sensors to control transport, waste, energy and water management [13]. Knowledge representation, access to information, and being able of processing and searching big data from heterogeneous sources are key factors to generate smart actions.

This paper presents a perception and control architecture for the management of a SHESS with the aim to reduce heating and cooling loads. In a preliminary work [14], an approach of a smart city model was proposed where semantic web technologies play a key role. Now, we delve into the nZEB where a semantic sensor network is deployed and ontologies are used, abstracting the terminology domain necessary to build a context-aware environment. The main goal is to describe sensors to improve the integration of the systems and the representation of knowledge about perceptions and

\footnotetext{
${ }^{1}$ http://purl.oclc.org/NET/ssnx/ssn
} 
observations of interest, in order to build a context-sensitive system using SWRL rules.

The rest of the paper is organized as follows. The proposed context-awareness architecture is presented in Section II. Section III deals with ontologies and semantic web technologies in which the semantic sensor model used in the proposed architecture is shown. Section IV describes a case of use and some SPARQL queries results obtained with the model and the SWRL rules. The paper ends with the conclusions.

\section{CONTEXT-AWARENESS ARCHITECTURE APPROACH.}

Several context definitions have emerged in the last decades, highlighting one or more primary elements to characterize the state of an entity: location, activity, identity name and time. Context is any type of information used to characterize the state of an entity, where entity is a person, building, sensor, even abstract activities. A system is contextawareness if context is used to provide relevant information and/or service to the user [15].

The components that form the context of the entity can be static or dynamic. It is clear that sensor nodes have static information such as the type and identity of the transducer. The user as an entity has static information in their profile but also dynamic information such as location in the building. User's location information can be get when logged to a wireless sensor node via mobile phones or by presence sensors. Thus, all data coming from world through sensors, database, and so on, are useful to identify situations such as user is at home or bus driver needs to change is current path among others [1619]. The real power of context-aware applications is that allow to puzzle out which sensors and data are required to reduce tasks or even to build applications invisible to the user [20].

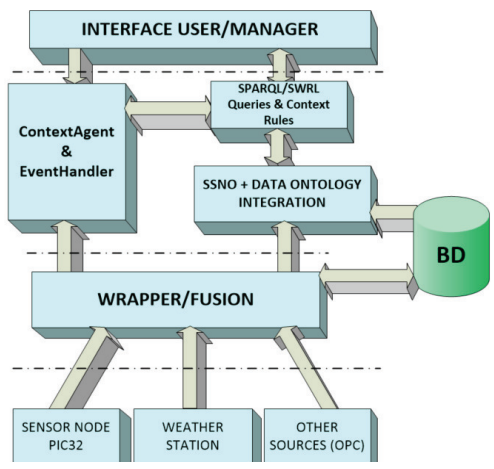

Fig. 1. Four layers context-awareness architecture.

The four layers of the context-awareness architecture here proposed are shown in Figure 1. Bottom layer is devoted to acquire data from sensors such as those coming from a CANBUS, WiFi, or any supported protocol by an OPC connection. Next layer, wrapper/fusion, performs data pre-processing or compute temperature averages to be stored. This layer builds some virtual sensors, such as a thermal flow virtual sensor. Here, some data emerge becoming part of the core ontology, and are ready to use for the next layer.

The second last layer has three major components working as a decision support layer. First one is built by some SSNO instances, set up from last sensor values coming from the wrapper/fusion layer. Here a data integration module is designed to retrieve information from database sources where data such as user profile, agenda, and building properties is stored. W3C owl time, DUL (DOLCE Ultralite) were Ontologies imported. Few changes were needed, one of these has to do with the elimination of the ontology DUL automatically imported because the version of the pellet engine reasoner is incompatible with this ${ }^{2}$.

Second component is about queries, and context rules. OWL-API is used to manipulate the ontology stored but also the protégé editor to graphically visualize the ontology. SPARQL queries allow users to extract information about nZEB state such as heat flows, through a user interface. $\mathrm{SWRL}^{3}$ contains logical rules already identified in the expert knowledge base and models to visualize the behavior of the building envelope. SWRL defines some special predicates called core built-ins, even it is possible to define a custom built-ins. SWRL rules are used and processed to execute actions for building a context-awareness smart building.

The last component, is event handler and a context agent. Event handler is used to identify a pattern in the data of the wrapper/fusion layer to guide the execution of a rule, and the context agent supports output results from context rules. Event handler interpreted sensor data in terms of event descriptions. Consider the following, when a user leaves an open window in a room, it changes surrounding thermal comfort conditions that are indicated by observed properties such as a temperature, and relative humidity [21]. We infer somebody as open a window. Context agent works like a parser among user interface, SSNO instances, and SWRL results. Thus when a SWRL rule is fired a command and a readable message for user interface is build such as "start flow flowing from solar collector south face to ground heat exchanger warm zone".

Finally, a user/manager interface allows interactions with the system. Here, a user is able to set up sensors, alarms and events limits, where some of these changes affect SWRL rules behavior. Developed sensor nodes are based in the PIC32 microprocessor with a web server so the user visualize information as a report through these nodes and eventually interact with them. This gives an additional user interface, anywhere inside the building, to access nZEB status or cancel any action. The following sections describe the key points of the four levels architecture.

\section{The Role of ONTOlogies AND SEMANTIC SENSORS IN SMART ENVIRONMENTS}

\section{A. Ontologies and Semantic Web Technologies}

Ontology definition by Gruber and Borst merged in [22], stating that: "An ontology is a formal, explicit specification of a shared conceptualization"

\footnotetext{
${ }^{2}$ http://lists.w3.org/Archives/Public/public-xgssn/2014May/0005.html

${ }^{3}$ The programming of this module was based primarily on http://dior.ics.muni.cz/ makub/owl/\#swrl
} 
In [23] conceptualization, shared, and formal and explicit specification is deeply explained. Here, conceptualization highlights important terminological aspects related to concepts and relations. Conceptual relations can be unary such as "temperature sensor" and "transducer" or binary such as "has transducer". "Temperature sensor" has just one subject such as "MBT3270 is a temperature sensor", similarly for transducer. On the other side, "has transducer" implies a subject and an object such as "MBT3270 has transducer NTC-05CA101K". Moreover, "formal and explicit specification" deals with the difficulty to express computational knowledge by not only including concepts but also axioms and properties. For instance, we can define that "has transducer" is asymmetric and intransitive, that means "MBT3270 has transducer NTC05CA101K" but "NTC-05CA101K has transducer MBT3270" is cause of an inconsistency. In this way, whenever we find something like "x has transducer $y$ " without any inconsistency, we can state " $x$ " is a kind of temperature sensor. When an ontology is build, such as SSNO, the key idea is to create carefully shared concepts, axioms and properties in a specific domain.

The semantic web idea is a web of data, a web of machine understandable information using semantic web technologies pointed out in "semantic web layer cake"4. Here, the resource description framework (RDF), RDF schema (RDFS) and Web ontology language (OWL) are three important specifications. $\mathrm{RDF}$ is a semantic data model for describing resources identified by something called Uniform Resource Identificator (URI). The main element of the model is the "sentence" or "triple", subject-predicate-object used to describe resource properties. RDF gives full freedom to declare even an absurd fact, because neither semantics nor the machine capacity can detect an error. RDFS goes further by describing class and properties to define terms and relations between them, with few inference capabilities. OWL is a description logic language with inference capabilities to represent terms and their relations. This offers a set of axioms to build an ontology, by providing explicit logical assertions about classes, individuals and properties and where it is possible to use inference engine or reasoners to infer other facts implicit into the ontology. The underlying structure in the semantic world is a collection of triples known as RDF graph and illustrated by a node and a directed-arc diagram, in which each triple is represented as a "node-arc-node" link and where each node points to another and so on. Because of that, a huge number of connections among nodes are usual so a query language is needed. SPARQL is a standard that appears in the semantic web layer cake as declarative query language for RDF based on HTTP. SPARQL has been the language that naturally access the graph structure and extracts the information accessed by a pattern that the user defines. This structure searches the user-requested pattern and if it matches any data structure, extracts the information and displays it to the user.

By using the existing software tools such as protégé, neon or topbraid composer, and using semantic web technologies, it's possible to model a domain by creating terms and define axioms and properties. These software tools and semantic web

\footnotetext{
${ }^{4}$ http://www.w3.org/2007/03/layerCake.svg
}

technologies have enough maturity and they are also used to populate, store, and visualize information. Even, they add reasoners such as pellet for questions and answering information. Ontologies and Semantic Web Technologies enable to build smart applications.

\section{B. Semantic Sensors}

Open Geospatial Consortium (OGC) is one of the leaders to solve the existing problem of "too much data due to a lot of sensors, but without enough knowledge". OGC members have specified, developed, and tested, interoperability interfaces and metadata encodings that enable integration of heterogeneous sensor webs into the information structure to be used by developers 5 .

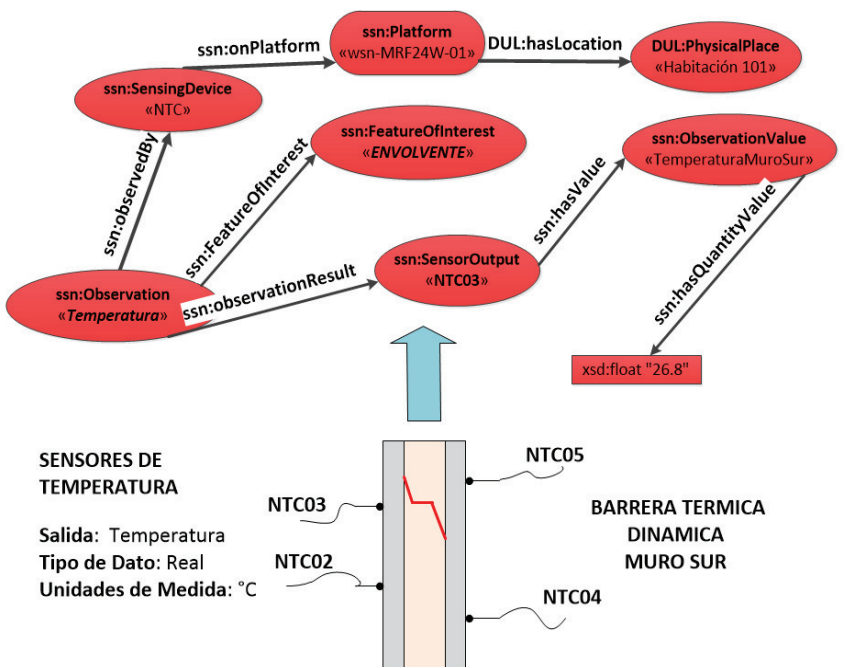

Fig.2. Sub-graph describing the "temperatureObservation" made by "NTC03" on January 15, 2013 for indoor wall temperature.

After OGC standards the W3C conducted a research on the use of ontologies and semantic web technologies to improve semantic interoperability and integrations in sensor networks. The SSNO is an OWL2 ontology that describes the capabilities and properties of sensors, the sensing action and the resulting observations [24]. Several applications have been deployed using the $\mathrm{SSNO}^{6}$ with the aim to break the gap between raw data and useful information where both user and machine exploit or consume in a better way. Figure 2, shows a part of the model by using SSNO to represent observations performed by the sensors node used after the wrapper/fusion layer. These kind of model give sufficient facts to answer some competence questions such as "find sensors where are located on south wall, and temperature values are greater than $20{ }^{\circ} \mathrm{C}$ ". Similarly, weather sensors were also used to populate the SSNO, and presence sensors where specific event of interest are "current location of a person".

A lists of question were used to determine the scope of the $\mathrm{SSNO}+$ Data Ontology Integration, such as, what events have

5

http://www.opengeospatial.org/projects/groups/sensorwebdwg

${ }^{6}$ http://www.w3.org/community/ssn-

cg/wiki/SSN_Applications 
happened? Here, SWRL rules are used to infer actions and express additional things. This gives end-user a powerful way to find how many times ground heat exchanger has been reloaded by solar collector. Moreover, it makes easy build an ontology, discovering classes, object properties and data properties needed. This SSNO is part of the smart building ontology which is also composed of $\mathrm{W} 3 \mathrm{C}$ owl time, building (materials, sub-systems -solar collector, ground heat exchanger, and thermal barrier-), and user (profile, and agenda) ontologies. Figure 3, shows on left-side some classes created where nZEBGroundHeatExchanger is pointed, upper right where this class is used, and lower right shows member of this class.

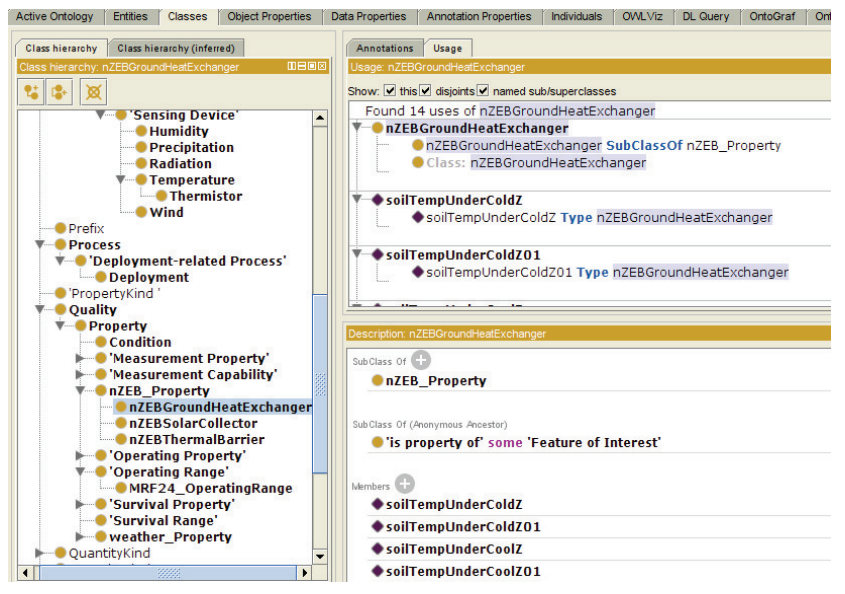

Fig. 3. Classes, and members for nZEB ontology.

\section{USE CASE AND EXPERIMENTS}

\section{A. $\quad n Z E B$}

The architecture proposal was deployed in a nZEB located at CAR, CSIC-UPM in Arganda del Rey, Madrid campus. A total of 45 sensors are installed to monitor indoor and outdoor variables such as presence, temperature and humidity, solar irradiation, underground and wall temperatures. The nZEB is a two story building, $10 \times 10 \mathrm{~m}$, which follows Isomax design [25]. It's composed of three renewable energy subsystems, roof solar collector, low enthalpy geothermal heat storage and thermal barriers, figure 4 .

- Solar Collector, is integrated in the roof of the building and is composed of a metal layer to capture solar energy. The metal layer has polypropylene (PP) tubes that adhere to the metal layer's inner face. PP tubes contain a water-glycol fluid, so the energy transferred from the metal layer to the fluid is conveyed through a hydraulic circuit to the ground heat exchanger for a thermal ground reloading or to the thermal barrier to reduce heating and cooling loads for thermal comfort. Pipe fluid temperatures reached on summer's days are close $70{ }^{\circ} \mathrm{C}$ and on winter nearly to 35 ${ }^{\circ} \mathrm{C}$ while environment is at 28 and $5^{\circ} \mathrm{C}$ respectively.

- Ground Heat Exchanger has four zones to store thermal energy, namely: Hot, Warm, Cool and Cold. The PP tubes from the hot and warm zones lay on a square area of $5 \times 5$ $\mathrm{m}$, concentric to the nZEB base. This area is completely isolated with expanded polystyrene (EPS). Average temperatures range from $18-22{ }^{\circ} \mathrm{C}$. The PP tubes of the cool zone lay in the remaining area under the perimeter of the house. The PP tubes of the cold zone cover the external perimeter of the house. Temperature values for cold and cool zones range from $12-17^{\circ} \mathrm{C}$. All polypropylene tubes are at $2 \mathrm{~m}$ ground depth except those at the hot zone which are at $3 \mathrm{~m}$.

- Thermal Barrier, it is integrated to reduce energy consumption in the heating and cooling of the nZEB. This is achieved by modifying the thermal gradient between the inside and outside temperature. Unlike normal constructions which have a temperature gradient that follows a pattern throughout the year, the nZEB can dynamically modify the gradient injecting a hot or cold fluid through the PP tubes on the exterior walls as shown in figure 5 .

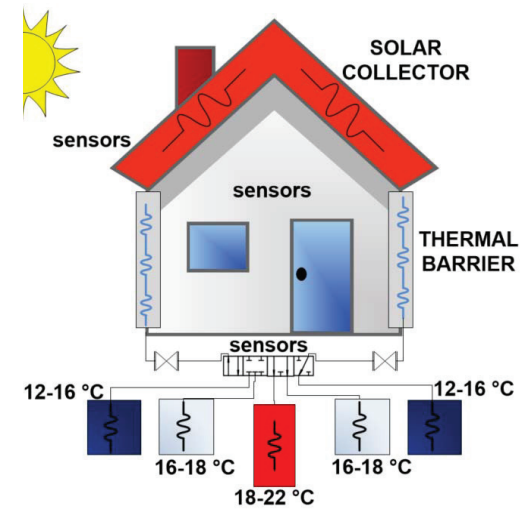

Fig. 4. nZEB renewable energy subsystems.

Sensors are installed in each of these systems for perception and monitoring to control the electro valves to set the path of the fluid.

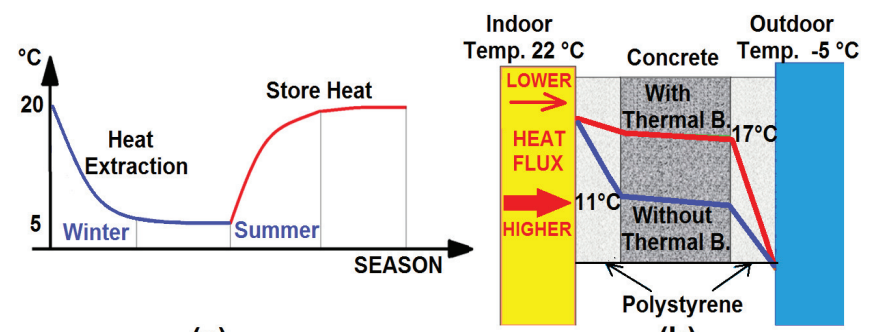

(a)

Fig. 5. (a) Ground Heat Exchanger, temperature variations. In summer time the heat energy is stored underground to be used in winter. (b) Thermal Barrier. Blue line shows temperature gradient between indoor and outdoor, and the red line the change in the temperature gradient by the heat fluid barrier.

\section{B. Sensor Network}

A sensor network is deployed for monitoring and control of the building, connected with the end user. A bus CAN is used to acquire all variables. The sensor nodes were developed at CAR and they are protected by a patent [26]. Sensors node is based on PIC32 microprocessor with bus CAN and Wi-Fi communications channels (figure 6). The TCP/IP stack for 
PIC32 provides a subset of HTTP methods (GET, PUT) and a light web server application. Because of that, default interface to show status or relevant information to user is performed through a mobile phone or any device with an Internet browser and Wi-Fi connection.

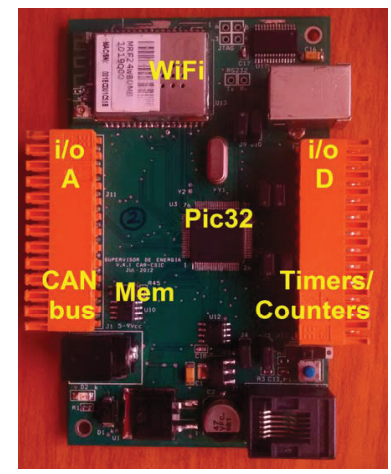

Fig. 6. The sensor node components.

\section{Experiments}

In current case, the entity context can be established with good accuracy based on data collected by sensor, observed activities, and known characteristics, to better adapt and respond for user benefit. Thus, it is possible to know indoor temperature and heat flow at any specific moment, but also people presence in the nZEB, and data and properties of the walls, and windows.

A query to the proposed ontology is displayed in figure 7 . This query is performed using protégé, shows the user interest in temperatures greater than $20^{\circ} \mathrm{C}$. Here, sensors located in the ground heat exchanger display such temperatures, table I. Under these conditions an action is fired to the valve to conduct the fluid flow from the ground heat exchanger zone to the thermal barrier.

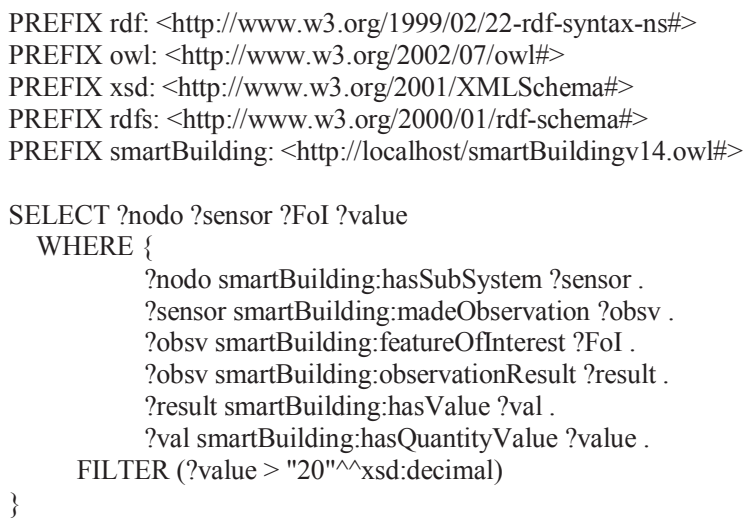

Fig. 7. SPARQL query to find sensors with temperature greater than $20^{\circ} \mathrm{C}$.

That means, temporal and spatial information exists as time is stored and sensor location inside building is identified. Exploiting this data by using SPARQL it is possible to calculate the period that a user remains in Room101 when room temperature is below $15^{\circ} \mathrm{C}$, even, ask where a person is located by specifying a temporal range for presence sensor.
This knowledge permits to weight decision to activate the thermal barrier by reducing heating and cooling loads from winter to summer. Here, semantic sensors give us useful information, Table II, but some logical rules are needed to build a context-awareness model.

TABLE I. SPARQL QUERY RESULTS

\begin{tabular}{|c|l|l|c|}
\hline node & sensor & FoI & value \\
\hline MRF24W_01 & NTC_Ch01 & soilTemperature & 20.6 \\
\hline MRF24W_01 & NTC_Ch02 & soilTemperature & 21.7 \\
\hline
\end{tabular}

TABLE II. SEMANTIC SENSOR INFORMATION

\begin{tabular}{|l|l|}
\hline \multicolumn{1}{|c|}{ Context } & \multicolumn{1}{c|}{ Triples } \\
\hline Location & Presence, highState, Room 101 \\
\hline Temperature & Room 101, hasTemp, $23^{\circ} \mathrm{C}$ \\
\hline Device & NTC04, model, "NCP18X" \\
\hline
\end{tabular}

A SWRL rule is extracted among others and displayed in Table III. As OWL language is not able to express all relations, SWRL is used to extend OWL expressivity. The SWRL rules are fired by events or by timing. The selection of the right valves for managing thermal energy flows depends on indoor temperatures, temperatures on each system -solar collector, ground heat exchanger, and thermal barrier-, heat flow on building façade, user presence, and even user activity, and weather conditions. Thermal comfort is quite different in summer or winter seasons, same for a user reading a book or doing exercise.

TABLE III. SWRL RULES FOR CONTEXT-AWARENESS

\begin{tabular}{|c|c|}
\hline & SWRL RULES \\
\hline 1 & $\begin{array}{l}\text { SensingDevice(?x1) } \square \text { hasFoI(?x1, ?roofTemp) } \square \\
\text { temperature(?x1, ?y1) } \square \text { swrlb:greatherThan(?y1, 25) } \square \\
\text { SensingDevice(?x2) } \square \text { hasFoI(?x2, ?IndoorTemp) } \square \\
\text { temperature (?x2, ?y2) } \square \text { swrlb:lessThan(?y2, 20) } \square \\
\ldots \\
\text { presenceSensor(?x2) } \square \text { hasValue(?x2, 1) } \square \\
\text { computedHeatFlux(?heatFlux) } \square \\
\text { swrlb: greatherThan (?heatFlux, 0.5) } \\
\rightarrow \\
\text { onPlatform(?x1, ?nodo) } \square \quad \text { solarCollector_valvesON(?nodo) } \\
\text { onPlatform(?x2, ?nodo) } \square \text { thermalBarrier_valvesON(?nodo) }\end{array}$ \\
\hline
\end{tabular}

Rule one shows an example where solar collector and indoor temperatures are acquired. Then using relational operators both temperatures are checked. While these temperatures are out of the indoor thermal comfort range but also heat flow is out of range, the thermal heat captured by the solar collector is ready to be sent to the thermal barrier. This is done by addressing the node id to the right valve to be opened.

A snippet from the eclipse environment where it is possible to access the third layer to make some queries and use pellet for inferences, is displayed in figure 8. SWRL rules are used and processed to execute actions for creating a contextawareness smart building. Moreover, the changes performed in 
the user schedule activities are detected as an event. For example, user is going back home at $4 \mathrm{pm}$ instead of $5 \mathrm{pm}$, then the event handler detects this change and a rule is fired to ensure that the thermal barrier will be on before user arrival.

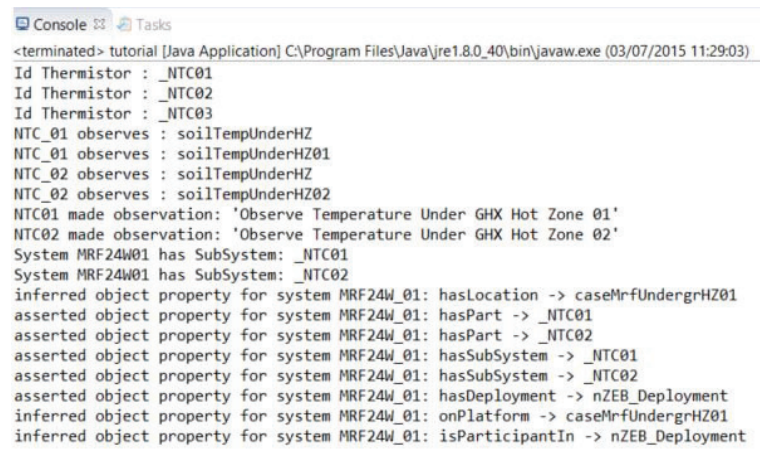

Fig. 8. OWL-API used for queries and discovering of inferred facts.

\section{CONCLUSIONS}

The present work shows some of the advantages of the context-awareness proposed that uses semantic web technologies and SWRL rules for: (i) the integration of heterogeneous data sources - databases, sensors, files- to increase the capabilities of the sensors, where the use of ontologies is a key factor; (ii) the management of the thermal energy in a nZEB building with automatic sensors data and knowledge representation; (iii) the modeling of contextual information and interrelationships.

The test on the nZEB demonstrates the ease of adding, deleting or changing SWRL rules to modify the behavior of smart building. The architecture developed allows quick editing and changing of the parameters of the rules as the data are semantically enriched and therefore it helps inexperienced users to better understand the system behavior. The contextawareness architecture proposed automatically decides what action to perform according to sensor measurements and context information. Thermal energy management is achieved using the developed model, which controls the thermal energy flow from the three energy subsystems of the building.

\section{ACKNOWLEDGMENT}

O. H. U. thanks to the Alternative Energies Research and Development Foundation for a pre-doctoral grant and also CONACYT-CIATEQ for additional economic support.

\section{REFERENCES}

[1] M. Beerepoot, "Technology roadmap. solar heating and cooling", OECD/IEA; Paris, 2012.

[2] M. Krzaczek, Z. Kowalczuk, "Thermal barrier as a technique of indirect heating and cooling for residential buildings", Energy and Buildings, vol. 43, pp. 823-837, 2011.

[3] W. Wang, Z. Tian, Y. Ding, "Investigation on the influencing factors of energy comsumption and thermal comfort for a passive solar house with water thermal storage wall”, Energy and Buildings, vol. 64, pp. 218-223, 2013.

[4] ECEEE, "Nearly zero energy buildings: achieving the EU 2020 target", European Council for Energy Efficiency Economy, Stockholm, Febraury 2011.
[5] T. Boermans, A. Hermelink, S. Schimschar, J. Grözinger, and M. Offermann, "Principles for nearly zero energy buildings - paving the way for effective implementation of policy requirements", BPIE, Executive Summary, pp. 5-21, November 2011.

[6] T. Berners-Lee, J. Hendler, O. Lassila, "The Semantic Web", Scientific American, pp. 29-37, May 2001.

[7] A. Soylu, P. De Causmaecker, "Embedded semantics empowering context-aware pervasive computing environments", IEEE, Symposia and Workshops on ubiquitous, autonomic and trusted computing, pp. 310317, Brisbane, QLD, July 2009.

[8] J. Vinagre, M. R. Wilby, B. Rodriguez, "EEOnt: An ontological model for a unified representation of energy efficiency in buildings". Energy and Buildings, vol. 60, pp. 20-27, 2013.

[9] F. Abanda, J. Tah, R. Keivani, "Trends in built environment semantic Web applications: Where are we today?", Expert Systems with Applications, vol. 40, pp. 5563-5577, 2013.

[10] D. Bonino, F. Corno, F. Razzak, "Enabling machine understandable exchange of energy consumption information in intelligent domotic environments", Energy \& Buildings, vol. 43, pp. 1392-1402, 2011.

[11] M. J. Kofler, C. Reinisch, W. Kastner, "An intelligent knowledge representation of smart home energy", World renewable energy congress, pp. 921-928, Linköping, 2011.

[12] H. Chen, T. Finin, A. Joshi, L. Kagal, F. Perich, D. Chakraborty, "Intelligent agents meet the semantic web in smart spaces", IEEE Internet computing, vol. 8, issue 6, pp. 69-79, 2004.

[13] EU, "Mapping Smart Cities in the EU", January 2014.

[14] O. Hernández, D. Guinea, M. Santos, "Semantic Sensors: A proposal from smart building to smart city model", $2^{\text {nd }}$ Workshop on semantic web and linked open data, Oaxaca, Nov 2014.

[15] G. D. Abowd, A. K. Dey, P. J. Brown, N. Davies, M. Smith, P. Steggles, "Towards a better undestanding of context and context-awareness", Springer, $1^{\text {st }}$ Int. symposium on handheld and ubiquitous computing, pp. 304-307, September 1999.

[16] L. Rodzina, S. Kristoffersen, "Context-dependent car navigation as kind of human-machine collaborative interaction", IEEE, Int. Conference on collaboration technologies and systems, pp. 253-259, May 2013.

[17] T. A. Cherfia, K. Barkaoui, F. Belala, "A BRS-based modeling approach for context-aware systems", IEEE, Int. Conference on embedded and ubiquitous computing, pp. 310-314, August 2014.

[18] R. V. Prasad Yerra, P. Rajalakshmi, "Context aware building energy management system with heterogenous wireless network architecture", IEEE Wireless and mobile networking conference, pp. 1-8, April 2013.

[19] S. N. Han, G. M. Lee, "Semantic context-aware service composition for building automation system", IEEE Transactions on industrial informatics, vol. 10, pp. 752-761, Febraury 2014.

[20] W. Mark, "The computer for the 21 st century", IEEE pervasive computing, vol. 1, pp. 19-25, 2002.

[21] A. Devaraju, W. Kuhn, C.S. Renschler, "A formal model to infer geographic events from sensor observations", Int. Journal of Geographical Information Science, Vol. 29:1, pp. 1-27, July 2014.

[22] R. Studer, V. R. Benjamins, D. Fensel, "Knowledge engineering: principles and methods", Data and knowledge engineering, vol. 25, pp. 161-197, March 1998.

[23] N. Guarino, D. Oberle, S. Staab, "What is an Ontology?", HandBook on Ontologies, pp. 1-17, Springer, 2009

[24] M. Compton, P. Barnaghi, L. Bermudez, R. Garcia, O. Corcho, S. Cox, et al., "The SSN ontology of the W3C semantic sensor network incubator group", Web Semantics: Science, services and agents on the WWW, Vol. 17, May 2012.

[25] E. D. Krecke, "Low Energy Building, Especially Self Sufficient ZeroEnergy House", DE Patent WO 2011042192 A2, April 2011.

[26] D. Guinea, E. Villanueva, M. C. Garcia-Alegre, D. M. Guinea, D. Martin, D. Rodríguez, O. Hernández, "Energy supervisor device in buildings", ES Patent 2380029 B1. March 2013. 